\title{
Sodium-coupled monocarboxylate transporter 1 interacts with the RING finger- and PDZ domain-containing protein PDZRN3
}

\author{
Yusuke Otsuka ${ }^{1,2,3} \cdot$ Tomomi Furihata $^{1} \cdot$ Kiyoshi Nakagawa $^{1} \cdot$ Yuta Ohno $^{1,2} \cdot$ Yoshie Reien $^{1} \cdot$ Motoshi Ouchi $^{2}$. \\ Hidefumi Wakashin ${ }^{1} \cdot$ Shuichi Tsuruoka ${ }^{3} \cdot$ Naohiko Anzai $^{1,2}$ (-)
}

Received: 9 October 2018 / Accepted: 27 April 2019 / Published online: 16 May 2019

(c) The Physiological Society of Japan and Springer Japan KK, part of Springer Nature 2019

\begin{abstract}
Sodium-coupled monocarboxylate transporter SMCT1 (SLC5A8) mediates monocarboxylate transport in the proximal tubule of the kidney. We have identified PDZK1 and PDZ domain-containing RING finger 3 (PDZRN3) as potent binding partners of SMCT1, which has a PDZ motif (Thr-Arg-Leu), by yeast two-hybrid screening and revealed that PDZK1 enhances the transport activity of SMCT1. In this study, we aimed to characterize the interaction between SMCT1 and PDZRN3 as well as to examine how PDZRN3 regulates SMCT1 function. An interaction between SMCT1 and PDZRN3 through the PDZ motif was observed in a co-immunoprecipitation assay and yeast two-hybrid assay. A transport assay showed that PDZRN3 abolished the enhancing effect of PDZK1 on nicotinate uptake via SMCT1. Our results suggest that SMCT1 interacts with PDZRN3 and that PDZRN3 may regulate SMCT1 function by interfering with the interaction between SMCT1 and PDZK1.
\end{abstract}

Keywords SMCT1 $\cdot$ PDZRN3 $\cdot$ Monocarboxylate $\cdot$ Transporter $\cdot$ PDZ protein

\section{Introduction}

Monocarboxylates (MCs), such as lactate and ketone bodies, play an essential role in energy metabolism. They are not only waste metabolites of glucose or fatty acid but also substrates for energy supply in the kidney [1-3]. To prevent loss of such important substrates, most of the MCs are reabsorbed in the renal proximal tubule after being freely filtered through glomeruli. However, MCs that are hydrophilic in an anionic form in plasma and urine cannot pass through lipid bilayers. Sodium-coupled monocarboxylate transporters (SMCTs) have been identified as transporters that mediate

\section{Electronic supplementary material The online version of this} article (https://doi.org/10.1007/s12576-019-00681-w) contains supplementary material, which is available to authorized users.

Naohiko Anzai

anzai@chiba-u.jp

1 Department of Pharmacology, Chiba University Graduate School of Medicine, 1-8-1, Inohana, Chuou-ku, Chiba 260-8670, Japan

2 Department of Pharmacology and Toxicology, Dokkyo Medical University School of Medicine, Tochigi, Japan

3 Department of Nephrology, Graduate School of Medicine, Nippon Medical School, Tokyo, Japan the first step in the reabsorption of MCs on the apical side [4]. There are two isoforms of SMCTs, SMCT1 (SLC5A8), a high-affinity and low-capacity transporter in the $S 3$ region of the proximal tubule [5, 6], and SMCT2 (SLC5A12), a low-affinity and high-capacity transporter in the S1 and S2 regions of the proximal tubule [7]. Despite the physiological importance of SMCTs, there is little information on the regulatory mechanism of SMCTs except for several reports about gene silencing of SMCT1 in various tumors [8-10] and inhibitory effects of insulin and SGK1 on SMCT1 [11].

In recent years, many PDZ (PSD-95, DglA, and ZO-1) proteins have been identified. A PDZ protein that has more than one PDZ domain is known to bind to a specific threeamino-acid sequence on a carboxylate terminus of its binding partner, a PDZ motif [11]. Three types of PDZ motifs have so far been identified: Class I domain Ser/Thr $-\mathrm{X}-\Phi$, Class II domain $\Phi-X-\Phi$, and Class III domain Asp/ Glu $-\mathrm{X}-\Phi(\mathrm{X}=$ any residue and $\Phi=$ hydrophobic residue $)$ $[12,13]$. PDZ proteins are known to regulate localization of binding partners or molecular signaling by an interaction with their binding partners through their PDZ motifs. PDZK1, which was identified in sequence analysis of renal hypouricemia patients [14], interacts with the PDZ motif of the urate/anion exchanger URAT1 (Thr-Gln-Phe) and increases urate uptake via URAT1 [15]. In addition to 
PDZK1, the sodium/hydrogen exchange regulatory factor (NHERF) family is known as a family of PDZ proteins in the kidney [16].

SMCT1 has seven transmembrane domains (with an extracellular amino terminus and an intracellular carboxyl terminus), and the C-terminus last tripeptide (Thr-Arg-Leu) corresponds to the Class I PDZ motif. Based on these structural features, it can be presumed that a certain PDZ protein binds to the C-terminus of SMCT1 in the kidney. Previously, utilizing the yeast two-hybrid screening, we have identified PDZK1 as a putative binding partner of SMCT1 in the human kidney, and found that interaction of PDZK1 with SMCT1 significantly facilitates its $[3 \mathrm{H}]$ nicotinate uptake [17]. On the other hand, in this yeast two-hybrid screening, we have also identified PDZ domain-containing RING finger 3 (PDZRN3) as another candidate of SMCT1 associated proteins. However, an interaction between SMCT1 and PDZRN3 has yet to be characterized.

PDZRN3 is a member of the PDZRN family. Members of this family except for PDZRN4L have a RING domain and multiple PDZ domains. PDZRN3, which has one RING domain and two PDZ domains, is a cytosolic protein that is distributed in the kidney, lung, heart, and other tissues [18]. PDZ proteins of the PDZRN family are known to work as ubiquitin E3 ligases and to regulate the expression of membrane proteins on the cell surface by promoting their endocytosis $[19,20]$. From these facts, we predicted that PDZRN3 interacts through its PDZ domain with the C-terminal PDZ motif of SMCT1. There is little information about the interaction between SMCT1 and PDZRN3 and it remains unclear how SMCT1 interacts with PDZRN3.

In the present study, we investigated whether SMCT1 interacted with PDZRN3 through the PDZ motif and how PDZRN3 regulates SMCT1.

\section{Materials and methods}

\section{Materials}

$\left[{ }^{3} \mathrm{H}\right]$ nicotinic acid $(50 \mathrm{Ci} / \mathrm{mmol})$ was purchased from American Radiolabeled Chemicals (St. Louis, MO, USA). Human SMCT1 cDNAs were gifts from Dr. Vadivel Ganapathy (Texas Tech University Health Sciences Center, Lubbock, TX, USA). A human PDZRN3 cDNA clone (NM_015009) subcloned into the pCMV6-entry vector, which contains the
FLAG tag sequence at the C-terminal, was purchased from OriGene Technologies (Rockville, MD, USA). Fetal bovine serum was purchased from Biowest (Strasbourg, France). Fugene 6 Transfection Reagent was obtained from Promega (Madison, WI, USA). Other substances were obtained from Nacalai Tesque (Kyoto, Japan).

\section{Plasmid construction}

The C-terminal 68 amino acids of wild-type human SMCT1 was generated by PCR using specific primers (Table 1) and cloned into the EcoRI and XhoI sites of pEG202 (bait vector), which was termed SMCT1-CTwt. Single PDZ domains of PDZRN3 were generated separately by PCR using specific primers (Table 1) and cloned into the EcoRI and XhoI sites of pJG4-5 (prey vector), which were termed PDZRN3 PDZ1 domain and PDZRN3 PDZ2 domain, respectively. The full-length coding sequence of human SMCT1 wild type, SMCT1wt, along with its mutant lacking the last three amino acids of SMCT1 (SMCT1-CTd3) were inserted into the pcDNA3.1 vector (Thermo Fisher Scientific) and the pEGFP-C2 vector for functional analysis and for immunocytochemical analysis, respectively.

\section{Cell culture and transfection}

Human embryonic kidney 293 (HEK293) cells were maintained in Dulbecco's modified Eagle's medium (DMEM) supplemented with $10 \%$ fetal bovine serum (FBS), penicillin (100 units $/ \mathrm{ml}$ ), and streptomycin $(100 \mathrm{mg} / \mathrm{ml})$ at $37{ }^{\circ} \mathrm{C}$ and in $5 \% \mathrm{CO}_{2}$. The cells were subcultured in a medium with $0.05 \%$ trypsin-EDTA solution (containing $137 \mathrm{mM} \mathrm{NaCl}$, $5.4 \mathrm{mM} \mathrm{KCl}, 5.5 \mathrm{mM}$ glucose, $4 \mathrm{mM} \mathrm{NaHCO}{ }_{3}, 0.5 \mathrm{mM}$ EDTA and $5 \mathrm{mM}$ HEPES; $\mathrm{pH}$ 7.2) and were used after 15-25 passages. Transient transfections were performed with Fugene 6 according to the manufacturer's instructions.

\section{Preparation of cell lysates and immunoprecipitation}

Twenty-four hours after transfection as described above, HEK293 cells were lysed with a buffer containing $250 \mathrm{mM}$ $\mathrm{NaCl}, 50 \mathrm{mM}$ Tris (pH 7.4), 1 mM EDTA, $1 \%$ Nonidet P40 and protease inhibitor cocktail (Pierce Biotechnology, Rockford, IL, USA). The lysate was centrifuged at 13,000 rpm for $5 \mathrm{~min}$ at $4{ }^{\circ} \mathrm{C}$ and then the supernatant was collected. The supernatant was immunoprecipitated with a rabbit
Table 1 Primers used in the vector construction for the yeast two-hybrid assay

\begin{tabular}{lll}
\hline & Forward & Reverse \\
\hline SMCT1-CTwt & 5'-cggaattcacaggaggaagaaaacag-3' & 5'-catctcgagtcacaaacgagtcccattg-3' \\
PDZRN3-PDZ1 & 5'-tagaattcgggaagcgcgagaagtc-3' & 5'-cggctcgagttacatgatatgttcaaagg-3' \\
PDZRN3-PDZ2 & 5'-tagaattcgccctcactaagatgtc-3' & 5'-cttctcgagttaagctgtgaattgcatgg-3' \\
\hline
\end{tabular}


anti-SMCT1 antibody (ab99064, Abcam, Cambridge, UK), a mouse anti-FLAG antibody (M185-3L, MBL, Woborn, MN, USA), or a rabbit anti-GFP antibody (G1544, SigmaAldrich, St. Louis, USA) using a Pierce Classic Magnetic IP/ CoIP Kit (Thermo Fisher Scientific, Waltham, MA, USA).

\section{SDS-polyacrylamide gel electrophoresis and immunoblotting}

Cell lysates prepared and immunoprecipitants as described above (20-40 $\mu \mathrm{g} / \mathrm{lane})$ were subjected to SDS-polyacrylamide gel electrophoresis followed by immunoblotting with 1:100-diluted antibodies, a mouse anti-PDZRN3 antibody (H00023024-B01P, Abnova, Taipei City, Taiwan) or a rabbit anti-SMCT1 antibody. A sheep anti-mouse IgG antibody and a donkey anti-rabbit IgG antibody (HRP-conjugated, GE Healthcare, Buckinghamshire, England, UK) were used for secondary antibodies. Immunoblotting was developed with an enhanced chemiluminescence reagent, Clarity Max Western ECL Substrate (BIO-RAD, Hercules, CA, USA), and visualized by ImageQuant LAS 4000 (GE Healthcare).

\section{Immunocytochemical analysis}

GFP-SMCT1wt and PDZRN3-FLAG were transfected into HEK 293 cells transiently as described above. At $48 \mathrm{~h}$ after transfection, the cells were fixed with $4 \%$ paraformaldehyde in PBS for $10 \mathrm{~min}$, permeabilized with PBS containing $0.1 \%$ Tween 20 for $10 \mathrm{~min}$, and blocked with 3\% BSA (Wako Pure Chemical Industry, Osaka, Japan) for $1 \mathrm{~h}$ at room temperature. Samples were incubated overnight at $4{ }^{\circ} \mathrm{C}$ with 1:1000-diluted primary antibodies, rabbit antiGFP antibody and mouse anti-FLAG antibody (MBL), and then with 1:500-diluted secondary antibodies, donkey antirabbit IgG (Alexa Flour 488, Thermo Fisher Scientific) and donkey anti-mouse IgG (CF 555, Nacalai Tesque), for $1 \mathrm{~h}$ at room temperature. After they had been mounted in Prolong Gold antifade reagent containing DAPI (Thermo Fisher Scientific), immunofluorescence microscopy was performed using LSM 780 confocal laser scanning microscope (Carl Zeiss Microscopy, Oberkochen, Germany).

\section{Yeast two-hybrid assay}

A yeast two-hybrid assay was performed with the LexAbased GFP two-hybrid system (Grow'n' Glow system, MoBiTec, Göttingen, Germany) according to the manufacturer's instructions utilizing a bait with the last 68 amino acids of SMCT1 and prey vectors with individual PDZ domains of PDZRN3.

\section{Nicotinate transport assay}

HEK293 cells were seeded in 24-well culture plates at a density of $1 \times 10^{5}$ cells/well. At $24 \mathrm{~h}$ after the cells had been plated, they were transfected with SMCT1wt, SMCT1d3, PDZK1, and/or PDZRN3 as described above. A total of $0.5 \mu \mathrm{g} / \mathrm{well}$ of the plasmid mixture $(0.166 \mu \mathrm{g}$ of each) was used for transfection. DNA amount was controlled using an empty plasmid. At $24 \mathrm{~h}$ after transfection, the culture medium was removed, and the cells were washed three times with serum-free Hanks' balanced salt solution (HBSS) containing $125 \mathrm{mM} \mathrm{NaCl}, 4.8 \mathrm{mM} \mathrm{KCl}, 1.2 \mathrm{mM}$ $\mathrm{KH}_{2} \mathrm{PO}_{4}, 1.2 \mathrm{mM} \mathrm{MgSO} \cdot 7 \mathrm{H}_{2} \mathrm{O}, 1.3 \mathrm{mM} \mathrm{CaCl} 2 \cdot 2 \mathrm{H}_{2} \mathrm{O}$, $5.6 \mathrm{mM}$ glucose and $25 \mathrm{mM}$ HEPES, $\mathrm{pH} 7.4$ and then preincubated in the same solution at $37^{\circ} \mathrm{C}$ for $10 \mathrm{~min}$. The cells were incubated in a solution with $5 \mathrm{nM}\left[{ }^{3} \mathrm{H}\right]$ nicotinic acid at $37{ }^{\circ} \mathrm{C}$ for $1 \mathrm{~min}$. The uptake was stopped by adding ice-cold HBSS, and the cells were washed two times with the same solution. The cells in each well were lysed with $0.5 \mathrm{ml}$ of $0.1 \mathrm{~N}$ sodium hydroxide. After adding $2.5 \mathrm{ml}$ of INSTA-GEL PLUS (PerkinElmer, Waltham, MA, USA), radioactivity was determined using a $\beta$-scintillation counter (Tri-Carb 2800TR, PerkinElmer) and normalized by cellular protein content measured by using the BCA protein assay kit (Thermo Fisher Scientific).

\section{Statistical analyses}

Uptake experiments were conducted three times, and each uptake experiment was performed in triplicate. Values are presented as means \pm standard error of the mean (SEM). Statistical significance was determined by Student's $t$ test or one-way analysis of variance (ANOVA) followed by Fisher's protected least significant difference (PLSD) test. Differences were considered significant at $p<0.05$.

\section{Results \\ Interaction between SMCT1 and PDZRN3 in an immunoprecipitation assay}

We performed an immunoprecipitation assay using HEK293 cells to examine an interaction between SMCT1 and PDZRN3. HEK293 cells were transfected with pcDNA3.1 (Vector) and PDZRN3 tagged with FLAG (PDZRN3FLAG). Proteins of endogenous SMCT1 were immunoblotted with an anti-SMCT1 antibody from a lysate of Vector and PDZRN3-FLAG (Fig. 1a). From a lysate of PDZRN3FLAG, proteins of SMCT1 were immunoprecipitated with an anti-FLAG antibody (Fig. 1a) and proteins of PDZRN3 
a

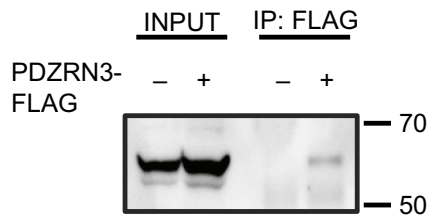

IB: SMCT1

b

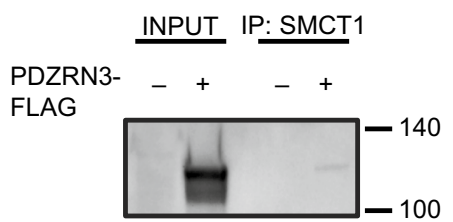

IB: PDZRN3

C

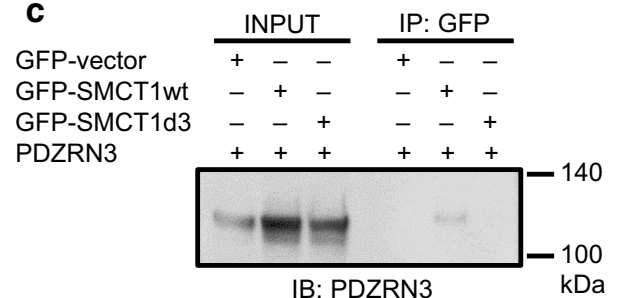

Fig. 1 Interaction between SMCT1 and PDZRN3 through the PDZ binding motif. HEK293 cells were transfected with FLAG-tagged pCMV6-entry or FLAG-tagged pCMV6-entry encoding the full length of PDZRN3. Cell lysates of these cells were co-immunoprecipitated with an anti-FLAG antibody (a) or with an anti-SMCT1 antibody (b). After SDS-PAGE, immunoblot analyses were performed with an anti-SMCT1 antibody (a) or with an anti-PDZRN3 antibody (b). HEK293 cells were transfected with PDZRN3 and pEGFP, GFP-fused SMCT1wt, or GFP-fused SMCT1d3, which is a mutant lacking the carboxyl terminal 3 amino acids from SMCT1. Cell lysates were co-immunoprecipitated with the anti-GFP antibody and immunoblotted with the anti-PDZRN3 antibody (c), $n=2$ were immunoprecipitated with an anti-SMCT1 antibody (Fig. 1b). These results indicate that there is an interaction between SMCT1 and PDZRN3.

Another immunoprecipitation assay was performed to confirm the interaction between SMCT1 and PDZRN3 and to investigate the role of the PDZ motif of SMCT1, TRL, at the carboxyl terminus for the interaction. HEK293 cells were transfected with PDZRN3 and pEGFP-C2 vector (VectorPDZRN3), GFP-fused SMCT1 wild-type (GFP-SMCT1wtPDZRN3), or GFP-fused SMCT1 mutant that lacked a PDZ motif (GFP-SMCT1d3-PDZRN3). Proteins of PDZRN3 were immunoprecipitated with the anti-GFP antibody from a lysate of GFP-SMCT1wt-PDZRN3 but not from a lysate of Vector-PDZRN3 or GFP-SMCT1d3-PDZRN3 (Fig. 1c). These results indicate that SMCT1 makes a complex with PDZRN3 and that the PDZ motif on the carboxyl terminus of SMCT1 is necessary for the interaction.

\section{Co-localization of SMCT1 with PDZRN3}

We performed immunocytochemical analysis to determine whether SMCT1 co-localizes with PDZRN3. SMCT1 fused with GFP at the amino terminus and PDZRN3 tagged with FLAG at the carboxyl terminus were transfected transiently into HEK 293 cells. After fixation by 4\% PFA, the cells were immunostained with anti-GFP antibody and anti-FLAG antibody or without primary antibodies (Fig. 2). Both proteins of SMCT1 (green) and PDZRN3 (red) were detected on the cell membrane, and a merge image showed that they co-localized (Fig. 2). This result indicates that SMCT1 and PDZRN3 co-localize.

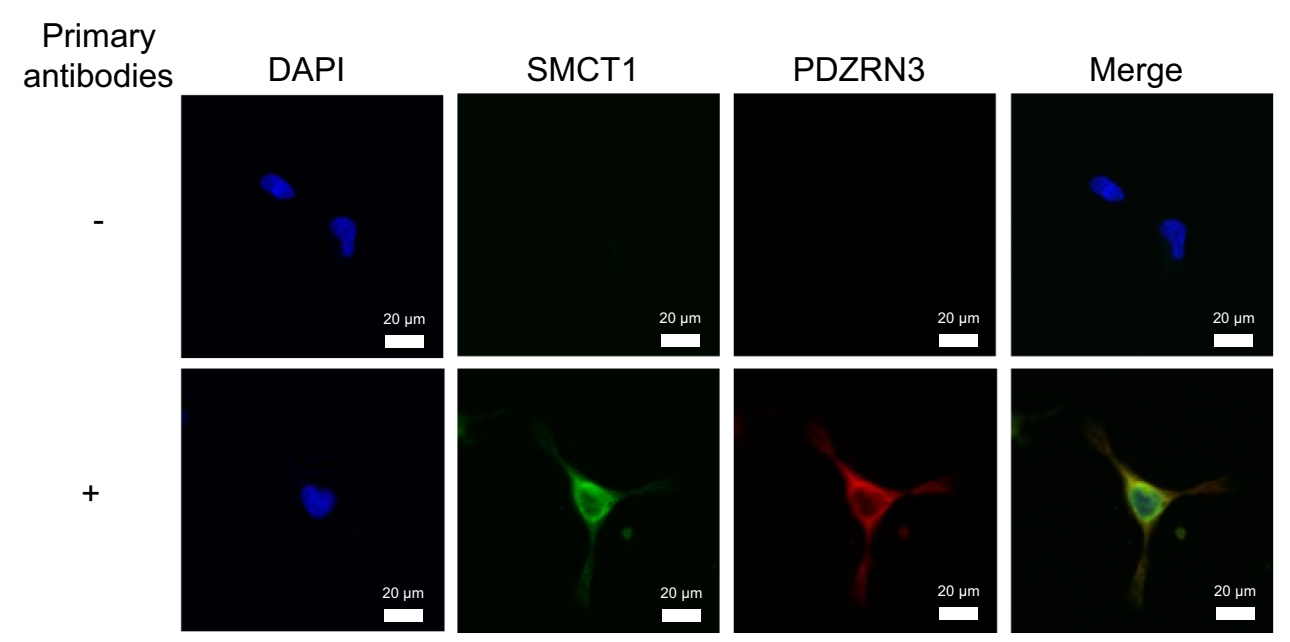

Fig. 2 Co-localization of SMCT1 and PDZRN3. Representative images of HEK293 cells transiently transfected with GFP-fused SMCT1 on the amino terminus and PDZRN3 tagged with FLAG on the carboxyl terminus. HEK293 cells were immunostained with a rabbit anti-GFP antibody and mouse anti-FLAG antibody followed by a goat anti-rabbit IgG conjugated with Alexa Flour 488 (green) and goat anti-mouse IgG conjugated with CF 555 (red) or stained only by secondary antibodies without primary antibodies. The nucleus was stained by DAPI (blue), $n=2$ 
Table 2 Specificity of PDZRN3 for interaction with SMCT1 in a yeast two-hybrid assay

\begin{tabular}{|c|c|c|c|c|c|}
\hline & \multirow{3}{*}{$\begin{array}{l}\text { C-terminal residues } \\
\text { (last } 6 \text { amino acids) }\end{array}$} & \multicolumn{4}{|c|}{ PDZRN3 } \\
\hline & & \multicolumn{2}{|c|}{ PDZ1 } & \multicolumn{2}{|c|}{ PDZ2 } \\
\hline & & LEU & GFP & LEU & GFP \\
\hline SMCT1-CTtwt & SNGTRL & + & + & - & - \\
\hline
\end{tabular}

A yeast two-hybrid assay was performed using the following bait and prey: bait with the last 68 amino acids of SMCT1 wild type (SMCT1CTwt) and prey containing the first PDZ domain of PDZRN3 (PDZ1) or the second domain (PDZ2). In the yeast two-hybrid assay, LEU2 and GFP were used as reporter genes instead of lacZ, which has been commonly used, because they enable detection of positive clones fast and easily with long wave UV. The results of a growth assay and GFP fluorescence are shown

\section{Domain analysis of PDZRN3}

We performed a yeast two-hybrid assay using the following vectors to examine the role of each PDZ domain of PDZRN3 in the binding. We constructed a bait vector containing the last 68 amino acids of SMCT1 (SMCT1CTwt) and also constructed two prey vectors with each of the PDZ domains (PDZ1 and PDZ2) of PDZRN3. In a yeast two-hybrid assay using these vectors, an interaction with SMCT1-CTwt was observed for PDZ1 domain but not for PDZ2 domain (Table 2). This result suggests an essential role of PDZ1 domain of PDZRN3 in the interaction with SMCT1.

\section{Transport activities of SMCT1 in the presence or absence of PDZRN3}

After confirming the interaction between SMCT1 and PDZRN3, we performed transport assays to examine the functional change of SMCT1 by its interaction with PDZRN3. We transiently transfected pcDNA3.1 containing full-length SMCT1 (HEK-SMCT1wt) or SMCT1 lacking the last three amino acids of its carboxyl terminus (HEK-SMCT1d3) or without an insert (HEK-mock) into HEK 293 cells in the presence or absence of PDZRN3. At $24 \mathrm{~h}$ after transfection, the cells were incubated with an uptake solution containing $5 \mathrm{nM}$ of $\left[{ }^{3} \mathrm{H}\right]$ nicotinate for $1 \mathrm{~min}$. The uptake of $\left[{ }^{3} \mathrm{H}\right]$ nicotinate in HEK-SMCT $1 \mathrm{wt}$ and HEK-SMCT1d3 was approximately 3.9-fold higher than that in HEK-mock. There was no significant difference in $\left[{ }^{3} \mathrm{H}\right]$ nicotinate uptake in HEK-SMCT1wt and HEK-SMCT1d3 regardless of whether PDZRN3 was transfected or not (Fig. 3). Transport activities of SMCT1 did not change regardless of whether PDZRN3 was transfected or not.

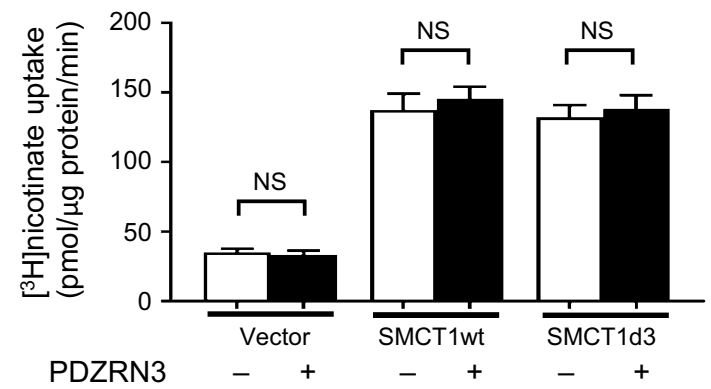

Fig. 3 Effect of PDZRN3 on $\left[{ }^{3} \mathrm{H}\right]$ nicotinate transport activity via SMCT1. To examine the functional change of $\left[{ }^{3} \mathrm{H}\right]$ nicotinate uptake via SMCT1 by co-expression with PDZRN3, HEK293 cells transfected with SMCT1 wild type (SMCT1wt) or a mutant of SMCT1 that lacks the last three amino acids (SMCT1d3) were incubated with an uptake solution containing $5 \mathrm{nM}$ of $\left[{ }^{3} \mathrm{H}\right]$ nicotinate for 1 min with or without co-transfection of PDZRN3. Then their radioactivity was measured. Each value represents the mean \pm SEM, $n=3$. Statistically significant $\left[{ }^{3} \mathrm{H}\right]$ nicotinate uptake was determined by Student's $t$ test or one-way ANOVA followed by Fisher's PLSD $(* p<0.05)$

\section{Cancellation effect of PDZRN3 on PDZK1-mediated SMCT1 activity enhancement}

For further examination of $\left[{ }^{3} \mathrm{H}\right]$ nicotinate uptake via SMCT1, we performed another uptake experiment using HEK-SMCT1wt additionally transfected with PDZK1 and with both PDZK1 and PDZRN3 or not. The uptake of $\left[{ }^{3} \mathrm{H}\right]$ nicotinate in HEK-SMCT1wt was approximately 3.7 -fold higher than that in HEK-mock. The uptake of $\left[{ }^{3} \mathrm{H}\right]$ nicotinate via HEK-SMCT1wt increased by 1.2-fold when PDZK1 was co-transfected. This enhanced effect of PDZK1 was abolished when PDZRN3 was additionally co-transfected (Fig. 4a). There was no significant difference in the uptake of $\left[{ }^{3} \mathrm{H}\right]$ nicotinate among HEK-SMCT1d 3 only, with cotransfection of PDZK1, and with co-transfection of PDZK1 and PDZRN3 (Fig. 4b). The uptake of $\left[{ }^{3} \mathrm{H}\right]$ nicotinate in HEK-SMCT1d 3 was not significantly difference from that in HEK-SMCT1wt. This transport assay showed that the augmentation of SMCT1 transport activity by PDZK1 was abolished when PDZRN3 was additionally co-transfected.

\section{Discussion}

We revealed the role of the PDZ motif in the interaction between SMCT1 and PDZRN3. The results of the immunoprecipitation assay showing that deletion of the PDZ motif from the SMCT1 carboxyl terminus abolished the interaction with PDZRN3 indicate that the PDZ motif of SMCT1 plays an essential role in the interaction between SMCT1 and PDZRN3. These results correspond to reports showing that the muscle-specific receptor tyrosine kinase (Musk) and Claudin16, which bind to PDZRN3, lose their 

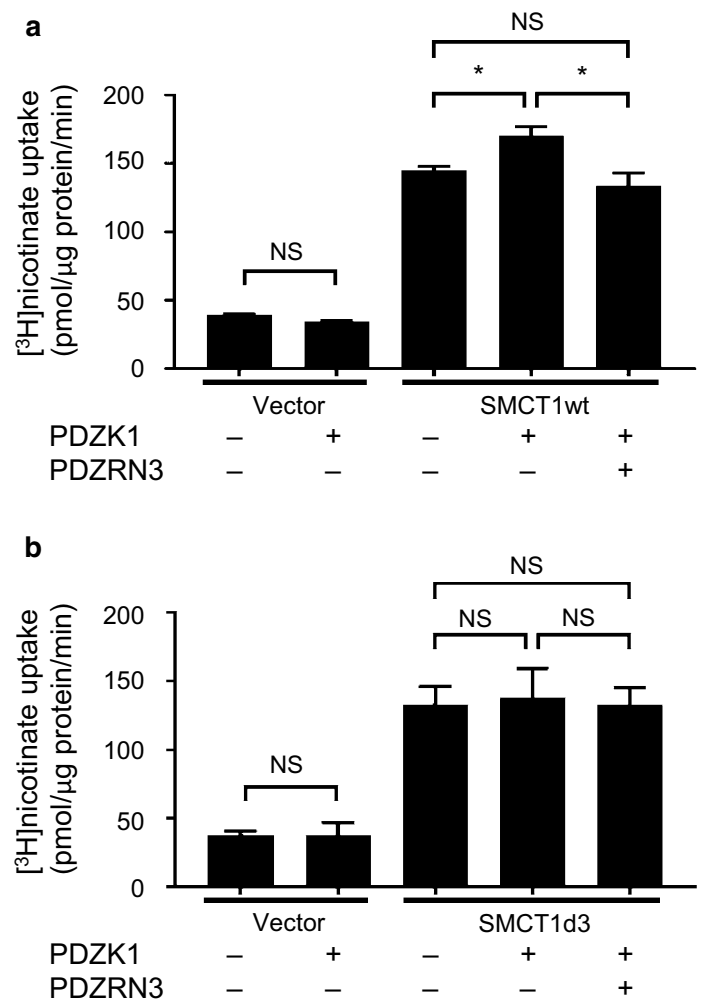

Fig. 4 Effect of PDZRN3 on $\left[{ }^{3} \mathrm{H}\right]$ nicotinate transport activity via SMCT1 enhanced by PDZK1. To examine the effect of PDZRN3 on the augmentation of SMCT1 uptake by PDZK1, $\left[{ }^{3} \mathrm{H}\right]$ nicotinate uptake via SMCT1wt (a) and SMCT1d3 (b) was measured when PDZK1 was co-transfected or PDZK1 and PDZRN3 were co-transfected as described in the legend of Fig. 3, $n=3$

binding ability when their PDZ motifs are deleted $[12,13]$. The necessity of the SMCT1 PDZ motif for interaction with PDZK1 has also been shown [17]. Based on these facts, SMCT1 interacts with PDZRN3 through its PDZ motif.

In addition to a role in binding, there is a PDZ motif has been reported to contribute to a function of its binding partner. Transport activities of proton-coupled peptide transporter 2, PEPT2, [21] and SMCT2 (unpublished data) decreased when their PDZ motifs were deleted. The PDZ motifs of PEPT2 and SMCT2 may contribute not only to binding to their binding partners but also to transport activity. However, there was no significant difference in nicotinate uptake via SMCT1 when PDZRN3 was co-transfected and not co-transfected (Fig. 3). This indicates that the PDZ motif of SMCT1 contributes to the interaction with PDZRN3 but not to its transport activity.

We also examined which of the two PDZ domains of PDZRN3 contributed to the interaction with SMCT1. There is one report about the specificity of the PDZ domains of PDZRN3. Musk in the neuro-muscular junction, which has a class I PDZ motif (Thr-Thr-Val) on the carboxyl terminus, interacts with PDZRN3. PDZ1 domain of PDZRN3 is essential for the interaction, but PDZ2 domain is not [12]. This corresponds to the results of the yeast two-hybrid assay in the present study showing that only PDZ1 domain of PDZRN3 bound to SMCT1. On the other hand, PDZ domains in a certain PDZ protein that contribute to binding have been reported to be different for each binding partner. At present, there is not sufficient information to determine the role of the specificity of PDZRN3 domains that contributes to a binding partner.

After confirming the interaction between SMCT1 and PDZRN3 through the PDZ motif, we examined the role of PDZRN3 in SMCT1 transport function. The results of the transport assay showing that uptake via SMCT1 did not change in the presence of PDZRN3 (Fig. 3) were unexpected. At first, we predicted that PDZRN3 negatively regulates nicotinate uptake via SMCT1 because PDZRN3, which is known to be a ubiquitin E3 ligase, regulates the endocytosis of Musk and CLDN16, which are membrane proteins that interact with PDZRN3 through their PDZ motifs $[12,13]$. However, it is not surprising that PDZRN3, which interacts with SMCT1, does not contribute to the functional change in consideration of an indirect effect of PDZRN3 abolishing the augmented effect of PDZK1 on SMCT1 function.

Uptake assays showed that PDZRN3 did not directly change the nicotinate uptake via SMCT1 (Fig. 3) and that it abolished the augmentation of SMCT1 transport activity by PDZK1 in the transport assay (Fig. 4). A putative mechanism may involve competition between PDZK1 and PDZRN3 in the interaction with SMCT1. There is a report showing that the cystic fibrosis transmembrane conductance regulator (CFTR) interacted with the CFTR-associated ligand (CAL), $\mathrm{Na}^{+} / \mathrm{H}^{+}$exchanger-3 regulatory factor 1 (NHERF1), and NHERF2 and that the relative affinity of these three PDZ proteins to CFTR may regulate CFTR endocytic recycling [22]. Although the affinity of PDZK1 and PDZRN3 to SMCT1 remains unclear, PDZRN3 probably competes with PDZK1 in binding to SMCT1 and interferes with the interaction between SMCT1 and PDZK1.

Regarding the role of SMCTs in absorption of MCs, there is a report showing that these two transporters play an essential role in renal excretion of lactate because lactate excretion into urine increased and serum lactate decreased in a mouse model (c/ebp $\delta$ null mice) that was deficient in expression of SMCTs only in the kidney [23]. Thus, SMCTs, which mediate the first step of the reabsorption of MCs in the renal proximal tubule, play an essential role in renal lactate excretion. However, the regulatory mechanism of SMCT1 is not fully understood. Our results showing that PDZRN3 abolished the enhancing effect of PDZK1 on SMCT1 function provide a clue for the mechanism of MC reabsorption via SMCT1. In addition, SMCT1 likely contributes to urate regulation by transporting anions that are exchange substrates for URAT1. It has been reported that uptake of uric acid via URAT1 is 
increased in the presence of SMCT1 [24]. This is probably because MCs in an anionic form that are transported into cells by SMCT1 make an outwardly-directed MC gradient and enhance urate transport via URAT1 [25]. Considering this linkage in urate transport between SMCT1 and URAT1, PDZRN3 may contribute to the regulation of uric acid.

In conclusion, the results of our study show that PDZRN3 interacts with SMCT1 through the PDZ motif on the carboxyl terminus and abolishes the enhancing effect of PDZK1 function. The results indicate that the interaction between SMCT1 and PDZRN3 is important because it may negatively regulate MC transport via SMCT1 by interrupting the interaction between SMCT1 and PDZK1.

Acknowledgements The authors would like to thank Asuka Morita and Masakatsu Nohara for their assistance in experiments. This work was supported in part by grants from the Japan Society for the Promotion of Science (KAKENHI 15590233, 18590900, 21390073, 26461258, 18K08200), Strategic Research Foundation Grant-aided Project for Private Universities (S1412001), The Nakatomi Foundation (2005, 2010), Gout Research Foundation of Japan (2008, 2009, 2010, 2011, 2012, 2013, 2015, 2016, 2017), Takeda Science Foundation (2007), the Salt Science Research Foundation (No. 0524, 0721), and Shimabara Foundation $(2006,2007,2013)$ to NA.

Author contributions NA designed the study. YO, YO and NA performed $\mathrm{Y} 2 \mathrm{H}$. NA, YR and $\mathrm{KN}$ checked and confirmed the experimental materials and data used in this study. YO, YO and MO performed transport assay. YO, YR, TF and HW performed biochemical and immunohistochemical analysis. YO, TF and NA wrote the manuscript. All authors discussed the results and commented on the manuscript.

\section{Compliance with ethical standards}

Conflict of interest All authors of this manuscript declare that we have no conflict of interest.

Ethical approval This article does not contain any studies with human participants or animals performed by any of the authors.

\section{References}

1. Guder WG, Wagner S, Wirthensohn G (1986) Metabolic fuels along the nephron: pathways and intracellular mechanisms of interaction. Kidney Int 29:41-45

2. Gerich JE, Meyer C, Woerle HJ, Stumvoll M (2001) Renal gluconeogenesis: its importance in human glucose homeostasis. Diabetes Care 24:382-391

3. Ross BD, Espinal J, Silva P (1986) Glucose metabolism in renal tubular function. Kidney Int 29:54-67

4. Garcia CK, Goldstein JL, Pathak RK, Anderson RG, Brown MS (1994) Molecular characterization of a membrane transporter for lactate, pyruvate, and other monocarboxylates: implications for the Cori cycle. Cell 76:865-873

5. Rodriguez AM, Perron B, Lacroix L, Caillou B, Leblanc G, Schlumberger M, Bidart JM, Pourcher T (2002) Identification and characterization of a putative human iodide transporter located at the apical membrane of thyrocytes. J Clin Endocrinol Metab $87: 3500-3503$
6. Gopal E, Fei YJ, Sugawara M, Miyauchi S, Zhuang L, Martin P, Smith SB, Prasad PD, Ganapathy V (2004) Expression of slc5a8 in kidney and its role in $\mathrm{Na}(+)$-coupled transport of lactate. J Biol Chem 279:44522-44532

7. Srinivas SR, Gopal E, Zhuang L, Itagaki S, Martin PM, Fei YJ, Ganapathy V, Prasad PD (2005) Cloning and functional identification of slc5a12 as a sodium-coupled low-affinity transporter for monocarboxylates (SMCT2). Biochem J 392:655-664

8. Deaton AM, Bird A (2011) CpG islands and the regulation of transcription. Genes Dev 25:1010-1022

9. Li H, Myeroff L, Smiraglia D, Romero MF, Pretlow TP, Kasturi L, Lutterbaugh J, Rerko RM, Casey G, Issa JP, Willis J, Willson JK, Plass C, Markowitz SD (2003) SLC5A8, a sodium transporter, is a tumor suppressor gene silenced by methylation in human colon aberrant crypt foci and cancers. Proc Natl Acad Sci USA 100:8412-8417

10. Thangaraju M, Gopal E, Martin PM, Ananth S, Smith SB, Prasad PD, Sterneck E, Ganapathy V (2006) SLC5A8 triggers tumor cell apoptosis through pyruvate-dependent inhibition of histone deacetylases. Cancer Res 66:11560-11564

11. Lopez-Barradas A, Gonzalez-Cid T, Vazquez N, Gavi-Maza M, Reyes-Camacho A, Velazquez-Villegas LA, Ramirez V, ZandiNejad K, Mount DB, Torres N, Tovar AR, Romero MF, Gamba G, Plata C (2016) Insulin and SGK1 reduce the function of $\mathrm{Na}+$ /monocarboxylate transporter 1 (SMCT1/SLC5A8). Am J Physiol Cell Physiol 311:C720-C734

12. Kim E, Sheng M (2004) PDZ domain proteins of synapses. Nat Rev Neurosci 5:771-781

13. Lee HJ, Zheng JJ (2010) PDZ domains and their binding partners: structure, specificity, and modification. Cell Commun Signal 8:8

14. Ichida K, Hosoyamada M, Hisatome I, Enomoto A, Hikita M, Endou H (2004) Clinical and molecular analysis of Patients with renal hypouricemia in Japan-influence of URAT1 gene on urinary urate excretion. J Am Soc Nephrol 15:164-173

15. Anzai N, Miyazaki H, Noshiro R, Khamdang S, Chairoungdua A, Shin HJ, Enomoto A, Sakamoto S, Hirata T, Tomita K, Kanai Y, Endou H (2004) The multivalent PDZ domain-containing protein PDZK1 regulates transport activity of renal urate-anion exchanger URAT1 via its C terminus. J Biol Chem 279:45942-45950

16. Cunningham R, Biswas R, Steplock D, Shenolikar S, Weinman E (2010) Role of NHERF and scaffolding proteins in proximal tubule transport. Urol Res 38:257-262

17. Sunena S, Nakagawa K, Xin H, Kimura T, Fukutomi T, Miyauchi S, Sakurai H, Anzai N (2019) Identification of the multivalent PDZ protein PDZK1 as a binding partner of sodium-coupled monocarboxylate transporter SMCT1 (SLC5A8) and SMCT2 (SLC5A12). J Physiol Sci. 69:399-408

18. Flynn M, Saha O, Young P (2011) Molecular evolution of the LNX gene family. BMC Evol Biol 11:235

19. Lu Z, Je HS, Young P, Gross J, Lu B, Feng G (2007) Regulation of synaptic growth and maturation by a synapse-associated E3 ubiquitin ligase at the neuromuscular junction. J Cell Biol 177:1077-1089

20. Marunaka K, Furukawa C, Fujii N, Kimura T, Furuta T, Matsunaga T, Endo S, Hasegawa H, Anzai N, Yamazaki Y, Yamaguchi M, Ikari A (2017) The RING finger- and PDZ domaincontaining protein PDZRN3 controls localization of the $\mathrm{Mg}(2+)$ regulator claudin-16 in renal tube epithelial cells. J Biol Chem 292:13034-13044

21. Noshiro R, Anzai N, Sakata T, Miyazaki H, Terada T, Shin HJ, He X, Miura D, Inui K, Kanai Y, Endou H (2006) The PDZ domain protein PDZK1 interacts with human peptide transporter PEPT2 and enhances its transport activity. Kidney Int 70:275-282

22. Cushing PR, Fellows A, Villone D, Boisguerin P, Madden DR (2008) The relative binding affinities of PDZ partners for CFTR: 
a biochemical basis for efficient endocytic recycling. Biochemistry 47:10084-10098

23. Thangaraju M, Ananth S, Martin PM, Roon P, Smith SB, Sterneck E, Prasad PD, Ganapathy V (2006) c/ebpdelta Null mouse as a model for the double knock-out of slc5a8 and slc5a12 in kidney. J Biol Chem 281:26769-26773

24. Lu Y, Nakanishi T, Tamai I (2013) Functional cooperation of SMCTs and URAT1 for renal reabsorption transport of urate. Drug Metab Pharmacokinet 28:153-158

25. Enomoto A, Kimura H, Chairoungdua A, Shigeta Y, Jutabha P, Cha SH, Hosoyamada M, Takeda M, Sekine T, Igarashi T, Matsuo
H, Kikuchi Y, Oda T, Ichida K, Hosoya T, Shimokata K, Niwa T, Kanai Y, Endou H (2002) Molecular identification of a renal urate anion exchanger that regulates blood urate levels. Nature 417:447-452

Publisher's Note Springer Nature remains neutral with regard to jurisdictional claims in published maps and institutional affiliations. 